\title{
An update on transsexuality
}

\author{
P Ginige, AS Malalagama
}

\section{Abstract}

Gender is a socially and culturally determined construct, which denotes a person's perception of being a male, female, both, or neither. It lies on a spectrum from binary gender to gender queer. Transgender (TG) refers to the broad spectrum of individuals who identify with a gender different from their gender at birth. The distress caused by any incongruency between the assigned and perceived gender is known as gender dysphoria (GD) and is classified under mental and behavioural disorders in the International Classification of Diseases-10 and Diagnostic and Statistical Manual of Mental Disorders-5. Current population estimates of TG people range from 0.17 to 1,333 per 100,000 . There are no general population studies for Sri Lanka (SL). Transsexuals face various forms of abuse, including sexual, physical and psychological abuse, most often due to transphobia. They have a high risk of engaging in transactional sex and hence a high risk of HIV infection. GD in adults is associated with an elevated prevalence of co morbid psychopathology, especially depression, anxiety disorders and suicidality. Management of GD individuals needs a multisectorial or multi element team approach. SL is making some progress in this direction, with the Ministry of Health having recently approved a Gender Recognition Certificate. However, all stakeholders should be involved in formulating management guidelines on transsexuality.

Key words: transgender, gender dysphoria, gender nonconformity, psychosocial problems, gender identity

SL J Psychiatry 2018; 9(2): 4-9

\section{Introduction}

Gender is a social construct that denotes a person's perception of being a male or a female. The term "sex" is biologically determined, based on determinants such as sex chromosomes, sex hormones and genitalia. However, both gender and sex interact with each other in the development of the concept of gender identity, which is defined as an individual's fundamental sense of being male or female (1).

The International Classification of Diseases $10^{\text {th }}$ version (ICD-10) entertains the broad term of gender identity disorder (GID), in which the defining feature is incongruency between the anatomical sex and gender identity. Transsexualism is considered under GID, and the term is used when an individual seeks or has undergone a social transition from male to female (MtF) or female to male (FtM), which usually involves treatment with hormones or surgery (2). The future ICD-11 has proposed the new term "gender incongruence" to describe gender identity issues (3).
In contrast to the previous versions of the Diagnostic and Statistical Manual of Mental Disorders (DSM), DSM-5 has tried to emphasize and respect the diversity of the role of gender, allowing provisions to consider genders beyond binary stereotypes (i.e., male or female). The DSM-5 uses the term gender dysphoria (GD) replacing GID. GD is a general descriptive term, and broadly encompasses many individuals with distress due to an incongruency between the experienced or expressed (desired) gender and one's assigned (born) gender; the discrepancy and the distress are key components of the diagnosis (4). This change in the DSM-5 is expected to facilitate reliable identification and service provision needs. The term is more specifically defined when used as a diagnostic category.

The experienced gender may be any alternative gender (not just the opposite sex) that is different from the born gender (4). Not everybody who has a discrepancy with the born sex and the desired sex, suffers due to it (i.e., not all with this discrepancy suffers from gender dysphoria); hence the notion of transgender (TG) is

This is an open-access article distributed under the terms of the Creative Commons Attribution 4.0 International License, which permits unrestricted use, distribution and reproduction in any medium provided the original author and source are credited. 
validated and reserved for individuals with significant transient or permanent cross gender identification, with no distress about the incongruency.

Like any other biological parameter, gender too lies on a spectrum, varying from agender (the individual identifies with no gender), androgyny (identification as male and female simultaneously), gender fluid (a fluctuating gender identity), to pangender (considers self as a member of all genders). Individuals with genders that do not conform to the binary division of male or female are described under the umbrella term gender queer (5). Diverse dispositions regarding perceptions of one's anatomical sex, desires, social roles, sexual relationships, where there are more than two principal categories is called gender pluralism, and this has existed throughout history (6). Genders not conforming to a binary structure, or which identifies a third gender has been reported from varying cultures, on every continent of the world. For example, native Americans identify a third or fourth gender. Intersex and transgender individuals are known as "hijra" live in India and Pakistan. South Sulawesi, Indonesia, identifies five genders (7). But over the years, economic transformation in South Asia, as well as political and religious factors, has led to 'cultural cleansing' and which in turn appears to have strengthened heteronormative gender binarism (6). The World Professional Association of Transgender Health (WPATH) urges the de-psychopathologization of gender nonconformity, emphasizing that it is a common and culturally-diverse human phenomenon that should not be judged as inherently pathological or negative (8).

The gender queer spectrum is still foreign to the Sri Lankan community and even to the medical fraternity. The socio-cultural influence on gender binarism and the political culture in Sri Lanka make the process of "coming out” for TG individuals, a challenge. Psychiatrists could play a major role in educating the community, reducing the stigma and facilitating the services rendered for TG individuals in this country.

\section{Prevalence of transsexuals}

A significant rise in the help seeking behaviour among GD individuals has been noted globally (9). Reasons may include dissemination of information, as well as increased communication between GD individuals through the internet and a better understanding and acceptance of the condition (10). A study in 2016 reported that $0.6 \%$ of U.S adults may identify as TG (11). Current population estimates of TG range from 0.17 to 1,333 per 100,000 (12). The Massachusetts Behavioral Risk Factor Surveillance Survey reports that $0.5 \%$ of the adult population aged 18 to 64 years identified as TG between 2009 and 2011 (12).

There are no general population studies for Sri Lanka. The first author has managed 71 transsexual patients from around the country since 2003 to August 2018, in a tertiary care clinic at Teaching Hospital Peradeniya (THP), in central Sri Lanka (Ginige et al, 2018, unpublished data). An analysis of the characteristics of these transsexual individuals show that one third were in the age range of 31-35 years. A majority, 69.0\%, of these transsexuals were female to male (FtM). The internationally reported prevalence is 1 in 30,000 natal males and 1 in 100,000 natal females, indicating a higher prevalence of MtF transsexuals, compared to FtM transsexuals (13). Although the findings from this clinic may not be generalizable to all of Sri Lanka, the reasons for the apparent higher prevalence of FtM transsexuals in Sri Lanka warrants further exploration, for example to examine if factors such as the sociocultural belief of males being privileged, play a role.

\section{Psychosocial problems encountered by transsexuals}

Various forms of sexual abuse, physical aggression and psychological abuse are faced by transsexuals (14). The national Transgender Discrimination Survey conducted by the National center for transgender equality, USA showed that gender non-conforming people face discrimination in every sphere of life including education, employment, family life, housing, identification documents, health care, insurance and the justice system (14). Finding a partner and engaging in satisfying and mutually pleasurable sexual relationships are often challenging issues. They have worries and concerns about their sexuality and intimate relationships. Transsexuals may experience that even the simplest of activities, such as reaching for daily needs and services (e.g., using a toilet in a public place) challenging. Their education may be disrupted and they could be isolated from the family and friends. They may be under qualified to find better paid jobs, which may contribute towards the increased risk of transsexuals engaging in risky behaviours, such as prostitution. Furthermore, transsexuals may engage in transactional sex in exchange for money for sex reassignment surgeries. A total of $26 \%$ of TG people had lost their jobs in the U.S in 2011 due to gender identity/ expression and $11 \%$ had engaged in sex work for an income (14). Transactional sex has increased the risk of HIV among TG people, compared to the general population (15). Stigma due to external transphobia (fear and rejection of, or an aversion towards, TG people), discrimination and gross deficiencies in legal recognition are some of the major barriers in seeking basic rights in society, including the right to health and specific treatment for GD.

TG individuals are more likely to engage in high-risk sexual activities, for example, MtF transsexual women may feel unempowered to instigate condoms use. The TG population remains severely underserved in relation to HIV, with only $39 \%$ countries reporting in the National Commitment and Policy (15-16). 
A survey carried out by the Human Rights watch in 4 districts in Sri Lanka, among TG and homosexual people between October 2015 and January 2016, report psychological, physical and sexual abuse experienced by TG individuals, and legal discrimination towards this group, with regards to identification documents, travel, health care and interaction with law enforcement authorities (17).

A descriptive study conducted at National Hospital, Sri Lanka in 2017 among TG people found that rejection by the family was the most common socio-cultural problem they faced (46.2\%). Other social issues included limitation of employment opportunities (41.8\%), difficulties in forming relationships (40.3\%), deprivation of privileges (40.3\%), stigma (37.3\%) and legal issues (31.3\%) [Siriwardena et al, 2017, unpublished data].

Another descriptive study of epidemiological characteristics of TG individuals conducted at Teaching Hospital Peradeniya, where TG clients were drawn from 8 provinces of the country, found that a majority of TGs could not complete legal transition (67\%) and sexual reassignment surgeries (75\%) [Ginige et al., 2018, unpublished data].

\section{Presentation}

The way TG people present varies between different age groups. The prepubertal assigned females may express their strong wish to be a boy or claim they are already boys. They express wishes to grow up to be a man; prefer boys' names, attire, accessories, hobbies, male friends and express the wish to be called and treated as a boy. They dislike and reject anything that implicate them as females, such as their sexual anatomy, menstruation, school uniform dress, make up and feminine toys and games. They often wear baggy clothes to hide their breasts or tape their breasts to flatten them. They express a strong desire for primary and secondary sexual characteristics of the desired gender. The prepubertal assigned boys similarly express a strong desire to be a female and strongly dislike any masculine features they may have.

Adults with GD usually, but not always, express a desire to get rid of the primary or secondary sexual characteristics and a strong will to acquire characteristics of the expressed gender. They adopt the behaviour, dressing/ grooming and mannerisms of the desired gender and feel uncomfortable if they are regarded or have to function in the society as members of their assigned gender.

\section{Presentation with psychiatric comorbidity}

GD in adults is associated with an increased prevalence of co morbid psychopathology, especially mood disorders such as depression, anxiety disorders and suicidal behaviours, and they may present with clinical features of these psychiatric co-morbidities (18). Depression among these individuals is often precipitated by stigma, shame and isolation by society. In a national online sample of 1,093 TG participants in the United States, $44.1 \%$ had clinical depression and 33.2\% had anxiety (19). Depression is the most common psychiatric disorder found among TG individuals, even in a multicentre study done in four European countries [20]. A descriptive study of epidemiological characteristics and management issues of transsexuals from a tertiary care clinic setting in Sri Lanka conducted at Teaching Hospital Peradeniya reported that nearly one fifth (19.7\%) had a comorbid psychiatric condition on presentation, and depression was the most common diagnosis (16.9\%); $2.8 \%$ had an anxiety disorder, $2.8 \%$ had mental and behavioural disorders due to alcohol use, while $1.4 \%$ had bipolar affective disorder [Ginige et al., 2018, unpublished data].

\section{Management}

The management of GD individuals needs a multisectorial or multi element team. The WPATH has described required Standards of Care (SOC), last revised in 2007, to provide clinical guidance for health professionals and to provide safe and effective pathways for individuals with GD to achieve lasting personal comfort with their gendered selves. Core principles laid out in the SOC include the following: Exhibit respect for gender nonconforming people (do not pathologize); provide care (or refer to knowledgeable colleagues) that affirms the person's gender identity and reduces the distress of GD, when present; become knowledgeable about the health care needs of TG people, including the benefits and risks of treatment options for GD; match the treatment approach to the specific needs of patients, and facilitate access to appropriate care; offer continuity of care; and be prepared to support and advocate for GD persons within their families and communities. The SOC identifies the differences in availability of resources in different settings and encourages the best available care amidst limited resources (8).

Though there is no major evidence base for the management steps of GD, based on the best available science and expert professional consensus, the following steps in management have been suggested.

\section{Assessment of gender dysphoria and any coexisting mental health issues}

The psychiatrist should take the lead role in the management of GD patients by assessing and coordinating care. It is the responsibility of the psychiatrist to diagnose and assess the severity of GD, by a detailed history and mental state examination, and formulate goals together with the patient and decide upon a management plan. 
The psychiatrist also completes the gender recognition certificate. Any comorbid psychiatric conditions should be diagnosed and treated.

Comorbidity does not necessarily preclude treatment of GD, but the presence of comorbidity may hinder the proper assessment and may adversely affect the capacity of the individual to give informed consent to important permanent treatments for GD. Thus, assessment of the patients' readiness for medical and surgical treatments is another important task of the psychiatrists. If the person is assessed to be ready, the psychiatrist would refer GD and TG individuals to relevant specialists such as endocrinologists, plastic surgeons, gynaecologists and urogenital surgeons. It is the responsibility of these relevant specialists to assess hormonal levels, internal sexual organs and perform general investigations prior to any surgical interventions $(8,21)$.

\section{Gender Recognition Certificate (GRC)}

GRC is an outcome of a campaign with a wider vision to recognize the needs of the individuals with GD in this country, carried out by 16 stakeholders under the leadership of the Human Rights Commission of Sri Lanka (HRCSL), the Sri Lanka College of Psychiatrists and the Ministry of Health (MoH). Much deliberation among these and other key stakeholders such as the Registrar General, the Inspector General of Police, the National STD/AIDS Control Program, the National Mental Health Institute, the Department of Registration of Persons, Department of Immigration and Emigration, non governmental organizations (NGOs) and most importantly representatives from the TG community, have resulted in the issue of a circular by the MoH in 2016, for all health institutions, setting out guidance on issuing the GRC $(17,22)$.

The GRC is the first legal document needed for a TG person to change their birth certificate and other important documents such as the identity card, educational certificates, passports and driving license, where the TG status and the perceived gender of the person is confirmed based on the comprehensive assessment by a psychiatrist. The certificate is issued to those above 16 years of age, and needs to be signed by the relevant psychiatrist and the Head of the institution (22).

\section{Psychotherapy}

Psychiatrists provide psychotherapy for issues related to GD, as well as education on treatment options, to patients, family members and partners. Psychotherapy during the process of change helps GD individuals by providing support, promoting resilience, and assisting in the development of interpersonal skills and coping strategies.

\section{Real-life experience (RLE) in the desired gender role}

"Living in a gender role that is congruent with one's gender identity" is called real life experience (RLE) (8). Varying techniques, such as voice training by speech and language therapists and drama therapy, could be innovatively used to achieve a successful RLE.

\section{Cross-sex hormone therapy}

Treatment with oestrogen or testosterone promotes the development of the secondary sex characteristics of the preferred sex, and partially suppresses features of the assigned natal sex (23). Hormone therapy is found to be associated with significant improvement in GD, psychological symptoms and comorbid conditions, quality of life, and sexual function in transsexuals (24).

\section{Sex reassignment surgery (SRS)}

SRS usually refers to feminizing or masculinizing genitoplasty (vaginoplasty, phalloplasty). Though some transsexuals remain at increased risk of psychiatric problems after SRS despite the relief of GD they usually experience, SRS is an effective treatment for GD and its outcomes are generally favourable (25).

Management of children and adolescents with GD include additional management principles where an extensive exploration is carried out on psychological, family, and social issues, in order to decide on fully or partially reversible or irreversible procedures; for example, administration of puberty supressing hormones [8]. Currently there are no child and adolescent gender clinics in Sri Lanka and so far no hormone therapy or SRS has been carried out locally in this age group. At present, the few specialists in this field in Sri Lanka validate the "stories" of the children and adolescents with GD, and provide education on the nature of the condition to families as well as patients, in order to minimize the distress and possible psychosocial harm, until the young person completes 18 years of age.

\section{The role of non-governmental organizations (NGOs) in promoting health in gender dysphoric individuals Sri Lanka}

In the absence of government involvement in the rights and cares of individuals with GD until recently, NGOs have been addressing this challenge for decades. The NGO Equal Ground has actively pioneered safeguarding the rights and reducing discrimination against the lesbian gay bisexual transgender (LGBT) community in Sri Lanka from 2004 (26). Other NGOs include Venasa, which was the first NGO formed for the benefit of the transsexual community alone in 2015, and the National Transgender Network, catering to the TG community, established in $2018(27,28)$. These organizations provide a forum for the marginalized TG community to share and care via 
counselling hotlines and networking, disseminating of information to create awareness, and conducting health campaigns, workshops and training.

\section{The way forward}

Asian countries including Nepal, India and Pakistan are now recognizing a third gender. Nepal's Supreme Court concluded that the ability to obtain documents bearing a third gender should be based on "self-feeling," and not the opinions of medical professionals or the courts (14). The introduction of the GRC and positive involvement of the $\mathrm{MoH}$ and the HRCSL in issuing a circular recommending starting services for individuals with transsexualism in every health institution with a psychiatrist, are promising developments in Sri Lanka (22). This move places Sri Lanka ahead of some other countries in the region, which still have little or no provisions to cater for individuals with GD, such as the Maldives.

Key areas that require further development in Sri Lanka include the establishment and collaboration between multi-element team members, conducting population studies to estimate the prevalence of TG individuals in the country, urging for non discriminative policies and anti-discrimination laws that explicitly include GD/TG individuals and formulation of local management guidelines. Raising awareness and education is needed in order to develop a TG friendly community. The formation of National Good Practice guidelines for Sri Lanka, through a collaboration between multi sectorial groups including psychiatrists, endocrinologists, gynaecologists, plastic surgeons, counselors, social workers, lawyers, committed NGOs, families of transsexuals and TG individuals is imperative. An attitudinal change in all relevant professional groups, and education of the public is essential to provide better care and standards of life for TG individuals in Sri Lanka.

\section{Acknowledgements}

The authors would like to gratefully acknowledge the assistance of Dr Evangalin Arulpragasam, Temporary Lecturer, Department of Psychiatry, Faculty of Medicine, University of Peradeniya.

P Ginige, Department of Psychiatry, Faculty of Medicine, University of Peradeniya

AS Malalagama, Base Hospital Diyatalawa

Corresponding author: AS Malalagama

Email: shakunthini@yahoo.com

http://orcid.org/0000-0002-5507-9815

\section{References}

1. American Psychological Association (APA). Answers to your questions about individuals with intersex conditions. Washington: APA; 2006.

2. World Health Organization (WHO). The ICD-10 classification of mental and behavioural disorders: clinical descriptions and diagnostic guidelines. Geneva: WHO; 1992.

3. World Health Organization (WHO). The International Classification of Diseases 11 $11^{\text {th }}$ Revision is due by 2018. Geneva: WHO; 2015. Available at: http://www.who.int/ classifications/icd/revision/en/

4. American Psychiatric Association: Diagnostic and Statistical Manual of Mental Disorders, Fifth Edition. Arlington, VA: American Psychiatric Association; 2013.

5. Madeline B. Guidelines for the primary and genderaffirming care of transgender and gender nonbinary people. San Francisco: Center of Excellence for Transgender Health; 2016.

6. Peletz MG. Transgenderism and gender pluralism in Southeast Asia since early modern times. Curr Anthropology 2006; (47)2: 309-40.

7. Tharp AN. Gender Spectrum Theory. West Haven, CT: University of New Haven 2016.

8. World Professional association for Transgender Health (WPATH). Standards of care. East Dundee, IL: WPATH: 2007. Available at: https://www.wpath.org/media/cms/ Documents/SOC\%20v7/SOC\%20V7_English.pdf

9. Zucker JK. Epidemiology of gender dysphoria and transgender identity. Sex Health. 2017; 14(5):404-411.

10. Zucker KJ, Lawrence AA. Epidemiology of gender identity disorder: Recommendations for the standards of care of the World Professional Association for Transgender Health. Int.J. Transgenderism 2009; 11(1): 8-18.

11. Flores AR, Herman JL, Gates GJ, et al. How many adults identify as transgender in the United States? Los Angeles, CA: The Williams Institute; 2016.

12. Meier SC, Labuski CM. The demographics of the transgender population. In: Baumle AK, eds. International handbook of the demography of sexuality: New York, NY: Springer; 2013.

13. Hoenig J, Kenna JC. The prevalence of transsexualism in England and Wales. Br J Psychiatry 1974; 124: 181-90.

14. Grant JM, Mottet LA, Tanis J. Injustice at every turn: a report of the National Transgender Discrimination Survey. Washington DC: The National Gay and Lesbian Task Force and the National Center for Transgender Equality; 2011.

15. Keuroghlian AS. Sexual Health among Transgender People. A program of the Fenway Institute. Boston, MA: National LGBT Health Education Centre; 2016.

16. Joint United Nations Programme on HIV/AIDS (UNAIDS), The GAP report. Geneva: UNAIDS; 2014. 
17. Human Rights Watch. All five fingers are not the same "Discrimination on Grounds of Gender Identity and Sexual Orientation in Sri Lanka”. Human Rights Watch: Colombo; 2016.

18. Haas AP, Eliason M, Mays VM, et al. Suicide and suicide risk in lesbian, gay, bisexual, and transgender populations: review and recommendations. J Homosex 2011; 58 (1): 10-51.

19. Bockting WO, Miner MH, Swinburne et al. Stigma, mental health, and resilience in an online sample of the US transgender population. Am J Public Health 2013; 103: 943-51

20. Heylens G, Elaut E, Kreukels B. Psychiatric characteristics in transsexual individuals: multi centre study in four European countries. Br J Psychiatry 2014; 204(2), 151-6.

21. Coleman E, Bockting W, Botzer M, et al. Standards of care for the health of transsexual, transgender, and gender nonconforming people (7th version). Int J Transgenderism 2012; 13: 165-232.

22. Ministry of Health. Issuing of gender recognition certificate for transgender community - Circular no. 01-34/2016. Ministry of Health: Colombo; 2016.
23. Hembree WC, Cohen-Kettenis P, Delemarre-van de Waal HA, et al. Endocrine Treatment of Transsexual Persons: An Endocrine Society Clinical Practice Guideline. J Clin Endocrinol Metab 2009; 94(9): 3132-54.

24. Murad M, Elamin M, Garcia M, et al. Hormonal therapy and sex reassignment: a systematic review and metaanalysis of quality of life and psychosocial outcomes. Clin Endocrinol 2010; 72(2): 214-31.

25. Smith Y, Van Goozen S, Kuiper A, et al. Sex reassignment: outcomes and predictors of treatment for adolescent and adult transsexuals. Psychol Med 2005; 35(1): 89-99.

26. Equal Ground (homepage on the internet). [updated 2018 Jan 1; cited 2018 Jun 8].Available at: http://www.equalground.org/

27. Venasa Transgender Network. (homepage on the internet). [updated 2017 Dec 1; cited 2018 Jun 8]. Available at: https://www.astraeafoundation.org/stories/venasatransgender-network/

28. National transgender Network Sri Lanka Facebook page. (homepage on the internet). [updated 2018 Jun 1; cited 2018 Jun 8]. Available at: https://www.facebook.com/ ntnsrilanka/ 